\title{
Harvard Step Test and Cooper 12 Minute Test on Improvement of Fitness Index (VO2 Max) in Basket Athletes in Pekalongan City
}

\author{
Andung Maheswara Rakasiwi ${ }^{1}$, Eko Budi Prasetyo ${ }^{2}$, Irine Dwitasari Wulandari ${ }^{3}$ \\ \{maheswaraandung@gmail.com ${ }^{1}$, hasan143173@gmail.com ${ }^{2}$, irenealmera@gmail.com ${ }^{3}$ \} \\ Pekalongan University, Pekalongan, Indonesia ${ }^{1,2,3}$
}

\begin{abstract}
Fitness index is to know the ability of VO2 max which includes cardiovascular and cardiorespiratory fitness. In the Pekalongan city basketball athlete, the problem with athletes is the athlete's fitness condition, high level of activity or training activities. The purpose of doing a fitness index is to find out the effectiveness of providing a Harvard step test and 12-minute cooper test on basketball athlete fitness index to prevent collapsing of athletes while exercising. Pre and post-test group design method of the fitness ability of the athlete's index with the Harvard step test and cooper test methods. Research Results using measurements that have been made obtained results on the measurement of fitness index using harvard step test and cooper test of significant value. With better or increased endurance will reduce the risk of collapse in athletes caused by the inability to accept the portion of the exercise or compete.
\end{abstract}

Keywords: Harvard Step Test, 12 Minute Cooper Test, Fitness Index

\section{Introduction}

Sport has become a necessity of life for humans, exercise, especially health sports will be able to maintain and improve the degree of human life. Humans without sports activities will experience a decrease the physical condition so they are susceptible to various diseases. Sports activities from time to time always experience development because it is very needed for human life. Achievement sports are sports that in practice require intensive and leveled training because there is a target for achievement. Sports achievements are strongly influenced by the elements of tactics, techniques and the quality of physical conditions, so their development always involves research from experts. Every sports athlete needs good physical condition. Endurance athletes must also be good so that they are able to accept every training load given to achieve maximum performance. Endurance is the ability of the body's organs to work within a certain period. Endurance in sports is the ability of athletes to fight fatigue during activities or work. The cardiovascular system, breathing and circulatory system af- 
fected and affects the quality of endurence systems. The factors that influence endurance are the maximum ability to meet oxygen consumption, which is characterized by $\mathrm{VO} 2 \mathrm{max}$. VO2 max is the maximum volume of $\mathrm{O} 2$ that is processed by the human body during intensive activities. This $\mathrm{O} 2$ max volume is a level of the body's ability, athletes who have high VO2max have good endurance and fitness [1].

Body fitness in an athlete is a major aspect that must be considered by an athlete, this fitness can be seen from the physical, psychological and functional athlete. When viewed the fitness component can consist of 2 aspects namely physical fitness and physical skills. Physical fitness includes cardiovascular endurance, muscle strength, muscle endurance, flexibility and body composition. While skills include speed, explosive power, balance, agility and coordination. Athletes need overall health conditions be able to give their full competitiveness. Imbalance in the body will certainly affect the performance of athletes when participating in competitions, one of which is the index fitness component.

The given exercise will respond to the increase in metabolism in the body as a result of increased activity carried out. A good response to the provision of proper training will be very beneficial for an athlete to be able to maintain stamina and avoid the risk of sports injuries. Cardiovascular response when doing exercise is influenced by the work of the heart which is increasing, this is marked by an increase in heart rate. Cardio respiration response will also increase according to the activities carried out, this is marked by an increase in pulse rate and the amount of breathing done [2].

Endurence of the body's organs in sports is the ability of athletes to fight fatigue during activities or work, endurance training is affected cardiovascular system, breathing and circulatory system. Therefore, the factors that influence endurance are the maximum ability to meet oxygen consumption, which is characterized by $\mathrm{VO} 2$ max. VO2 max is the maximum volume of $\mathrm{O} 2$ that is processed by the human body during intensive activities. This $\mathrm{O} 2$ max volume is a level of the body's ability expressed in liters per minute or milliliters / minutes / kg body weight. Athletes who have high VO2max have good endurance and fitness [4].

For an athlete, fitness will be very much needed as an effort to achieve achievement targets, therefore providing proper training on the endurance of cardiorespiration, cardiovascular and flexibility of muscles as one of the main movers of the body. The training environment or competition will have different metabolic effects on the body. The training ground environment in the highlands or the cold environment or the training environment in the lowlands hot environment gives different effects on the body's metabolism. The body needs to adjust to the exercise activities carried out on the differences between the two environments. An athlete doing a competition in a hot environment will certainly accelerate metabolism in the body, spending more sweat and accompanied by a large amount of energy used so that the physical condition of an athlete will get tired more easily. If an athlete fights with a cold air environment will also have different effects on the body's metabolism. The heating is done and the process of burning the body's metabolism will be slow, energy will not quickly escape from the body [5].

Muscle, heart, cardiovascular or cardiorespiratory endurance is needed for athletes so that when athletes do exercises or compete in hot or cold environments it will make it easier for the body to adapt. So it will not reduce the risk of injury when competing or training.Seeing this, it is very necessary to measure the cardiovascular work 
ability of an athlete. If the athlete has good quality cardiovascular endurance, then the risk of physical fatigue and heart collapse when the athlete does the exercise or compete will decrease. Measurement and improvement of cardiovascular endurance in accordance with the type of exercise practiced by an athlete. Therefore, researchers are interested to know the benefits of the Harvard step test and cooper test for increasing endurance athletes in terms of the fitness index. This research will provide information for an athlete, agency manager, athlete trainer to be able to pay attention to athletes in terms of cardiovascular endurance and endurance to the activities of an athlete. Muscle, cardiovascular or cardiorespiratory endurance is needed for athletes so that when athletes do exercises or compete in hot or cold environments it will make it easier for the body to adapt. So it will not reduce the risk of injury when competing or training.

The cardiovascular system is a blood circulation organ consisting of the heart, blood components and blood vessels that function to deliver and supply oxygen and nutrients throughout the body's tissues that are needed in the body's metabolic processes. The cardiovascular system requires many varied mechanisms so that the regulatory function can respond to bodily activities, one of which is to increase blood supply activity so that tissue activity can be fulfilled. In severe circumstances, the blood flow is more directed at vital organs such as the heart and brain which function to maintain and maintain the circulatory system itself. Physical exercise is an activity a person does to improve or maintain physical fitness. Physical exercise is generally grouped into several categories, depending on the effect it has on the human body. Exercise results in physiological changes in almost all body systems, especially in the muscular and cardiovascular systems. The effect of exercise on the body is everything that happens in exercise. However, if the exercise load is too light, the effects of the exercise after recovery will be less than expected. If the training load is too heavy then the condition will return to normal. When an athlete does aerobic exercise, the heart and respiratory muscles become stronger. Also, blood pressure decreases athlete, and the number of blood cells increases. The body becomes more efficient and, as a result, exercises that were previously very heavy to become easier and add less weight to the body. Exercise becomes easier, so their ability to improve is reduced by overall fitness athletes [6].

Aerobic changes that occur in the aerobic system after exercise, namely increasing the content of myoglobin, the content of myoglobin in skeletal muscle has been shown to substantially increase the quality of training. Myoglobin is an oxygenbinding pigment that is similar to hemoglobin. In this case, it acts as a store for oxygen. However, this is considered a small function in contributing to the improvement of the aerobic system. Its main function is in helping the delivery (diffusion) of oxygen from the cell membrane to the mitochondria where it is consumed. Increased carbohydrate oxidation (glycogen). Training increases the capacity of skeletal muscle produce aerobic energy is increased. Evidence for this change is an increase in maximal aerobic power (VO2 max) [7].

Blood and heart circulatory system, the main changes that result from clear training at rest are changes in heart size, decreased heart rate, increased stroke volume, increased blood volume and hemoglobin and changes in skeletal muscle. Changes during maximum training are common knowledge that physical training greatly increases maximum work capacity. Some of the physiological changes needed to bring 
these improvements are an increase in maximal aerobic power, Increased cardiac output, Increased stroke volume, No change or slight decrease in heart rate, Increased lactic acid production, No changes in muscle blood flow.

Respiratory changes, ventilation is not a factor that can affect VO2 max, the increase in maximal ventilation must be considered when increasing VO2 max. The increase was caused by an increase in both tidal volume and respiratory rate. Training causes an increase in ventilation efficiency. Higher ventilation efficiency means that the amount of air ventilation at the same level of oxygen consumption is lower than in trained people. Because oxygen demand increases, it will also cause increased ventilation or air requirements in the lungs as a process of adaptation to greater oxygen demand. Some examples of activities or long activities such as running a marathon. oxygen to the respiratory muscles will be smaller than the need for oxygen in the skeletal muscles. Pulmonary volume can be measured in resting conditions (with the exception of tidal volume). Most of these changes can be attributed to the fact that training results can have a good effect on lung endurance. Athletes tend to have greater diffusion capacity at rest and during training than non-athletes. Diffusion of capacity per second is not directly affected by training. Greater lung volume in athletes will provide greater anatomy to form the alveolar and capillary capillaries.

\section{Methods}

This research is an experimental study with the research design used is quasi experiment design with pre and post-test group design. The population of this research is the basketball athletes KONI Pekalongan with research subjects as many as 30 people with the number of athletes aged 20 years 6 people, 21 years 12 people, 22 years 4 people, 23 years 2 people, 24 years 6 people. The early activity of this study was by asking permission to conduct research to the chairman of the KONI and the Chairperson of the Pekalongan Basketball Association as the person in charge as well as the field supervisor. Conducted socialization about the research to be carried out to KONI basketball players in Pekalongan City. Explain the purpose of the research and the procedures for conducting the research to be carried out by basketball coaches and athletes. The researcher sets the subject according to the inclusion and exclusion criteria set by the researcher. Sampling selecting athletes who represent the criteria, which have been determined by the inclusion criteria and the exclusions discussed in the eligibility criteria. Inclusion criteria included age, KONI Pekalongan athletes, willing to sample and sign informed consent, subjects with no history of physical abnormalities, subjects without cardiovascular or cardiovascular disease, patients with no structural abnormalities in posture, no other abnormalities were found. Exclusion criteria, namely subjects had posture abnormalities, subjects had a history of cardiovascular or cardiovascular disorders, a history of trauma to the vertebrae. The drop out criteria include subjects not being cooperative and not fulfilling the scheduled training program. Subjects during the study did not regularly follow the research procedures.

Data analysis includes several tests, namely the Fitness Index score comparison test between before and after training on the Harvard Step Test using a nonparametric comparison test, with the Wilcoxon match pair test. This test aims to de- 
termine the Fitness Index score after the Harvard Step Test. The significance level used is $\alpha=0.05$. Fitness Index score comparison test data between before and after training on the cooper test using the non parametric comparison test, with the Wilcoxon match pair test. This test aims to determine the Fitness index score after cooper test training. The significance level used is $\alpha=0.05$. Fitness index score comparison test before training in the two groups using the parametric comparison test. This test aims to compare the average Fitness Index scores of the Harvard Step Test and Cooper Test groups. The significance level used is $\alpha=0.05$.

\section{Results and discussions}

Subject characteristics include age, sex, description of the research subjects are presented in the following table.

Table 1. Characteristic of subject

\begin{tabular}{rlrrrr}
\hline & & Frequency & Percent & Valid Percent & Cumulative Percent \\
\hline \multirow{6}{*}{ Valid } & 20 & 6 & 20.0 & 20.0 & 20.0 \\
& 21 & 12 & 40.0 & 40.0 & 60.0 \\
& 22 & 4 & 13.3 & 13.3 & 73.3 \\
& 23 & 2 & 6.7 & 6.7 & 80.0 \\
& 24 & 6 & 20.0 & 20.0 & 100.0 \\
\cline { 2 - 6 } & Total & 30 & 100.0 & 100.0 & \\
\hline
\end{tabular}

From the table the number of subjects measured from male and female athletes in basketball is 30 athletes with several ages from the age of 20 to 6 people, 21 years 12 people, 22 years 4 people, 23 years 2 people and 24 years 6 people.

\subsection{Pre test and post test harvard step test}

On the results of the measurement to see differences in the results before the exercise and after the exercises are carried out can be said to have a significant difference if the p-value (p) obtained is smaller than 0.05 ( $p<0.05)$. Based on the analysis, the $t$-count value of -6.552 with a probability value of $0.000<0.05$ shows that there is a significant result between pre-test and post-test in the Harvard Step Test. So it can be concluded that administering the Harvard Step Test can improve the basketball athlete fitness index. 


\subsection{Pre test and post test cooper test}

On the results of the measurement to see differences in the results before the exercise and after the exercises are carried out can be said to have a significant difference if the p-value (p) obtained is smaller than $0.05(\mathrm{p}<0.05)$. Based on Table pre and post test the value of $t$-count is -6.318 with a probability value of $0.000 \mathrm{p}$ value $<0.05$, this shows that there are significant results between pre-test and post-test on the cooper test. So it can be concluded that administering the Cooper Test 12 minutes can improve the basketball athlete fitness index.

\subsection{Effects of Harvard step test and cooper test on Fitness Indeks}

The cardiovascular system performs work or activities to the maximum to pump blood and deliver oxygen to the muscles that contract, the release of adrenaline and lactic acid into the blood will increase heart rate. Sports activities will increase the work of several different components of the cardiovascular system. during the activity the skeletal muscle will increase cardiac output of the heart and blood flow from organs and inactive tissue will be redistributed to the active skeletal muscle. Skeletal muscle during exercise will increase cardiac output of the heart and blood flow from organs and inactive tissue will be redistributed to the active skeletal muscle. The body's response to physical stress carried out gives an increase in Heart Rate, Blood Pressure, Stroke volume, cardiac output, ventilation and VO2 max. Heart rate is controlled by two nervous systems, namely parasympathetic and sympathetic. Parasympathetic nerves secrete ACh and decrease heart rate, whereas sympathetic nerves release norepinephrine and increase heart rate. At rest, sympathetic and parasympathetic nerve stimulation is in a balanced state. During exercise, parasympathetic stimulation decreases and sympathetic stimulation increases. Stroke volume (SV) is controlled by the final diastolic volume, average aortic blood pressure and the strength of ventricular contractions. The final diastolic volume $=$ if the final diastolic volume increases, $\mathrm{SV}$ also increases. As the final diastolic volume increases, light stretching of the heart muscle fibers will increase the strength of the contractions. [1,8].

During sports activities blood flow also increases by decreasing the resistance of blood vessels to the systemic circulation of active skeletal muscle. Changes in oxygen delivery to the muscles during cardiac exercise increase with intensity until the maximum VO2 is reached. During exercise, general vasodilation arises due to accumulation of vasodilatory metabolites. This results in decreased peripheral resistance, which in turn, strongly increases sympathetic activity through baroreceptor activation. Increased sympathetic activity causes vasoconstriction in the visceral organs, where vasodilation is predominantly in muscle blood vessels and coronary circulation due to local vasodilatory metabolites. Cutaneous blood vessels initially respond to sympathetic activity with vasoconstriction. As exercise progresses, reflex temperature is activated and causes cutaneous vasodilation to reduce heat production by muscle activity $[4,9]$.

In this study the Harvard Step Test and Cooper Test methods provide cardiovascular adaptation responses due to structured and always increasing exercise, so that with increased training and activity will provide an increase in the fitness index of athletes. Many factors contribute to aerobic fitness, including the maximum capacity of the respiratory and cardiovascular system, a larger heart, red blood cells and more hemoglobin [10,11]. Genetic influence on muscle strength and muscle endurance is 
generally associated with the composition of muscle fibers consisting of red and white fibers. Someone who has more is more appropriate to do activities that are aerobic, while those who have more white skeletal muscle fibers, are better able to do activities that are anaerobic. Age affects almost all components of freshness. Cardiovascular endurance shows a tendency to increase in childhood up to around twenty years and reach a maximum at the age of 20 to 30 years [12]. This endurance will decrease with age, with a decrease of $8-10 \%$ per decade for inactive individuals, whereas for active individuals the decline is $4-5 \%$ per decade [5,11]. Cardiovascular endurance at the age of children, between men and women is not much different, but after puberty there are differences. The average young woman has aerobic fitness between 15-25\% smaller than young men and this depends on their level of activity. But in young women athletes who often practice only differ $10 \%$ below male athletes of the same age in terms of VO2max. Women have $27 \%$ fat tissue from body composition more than men $15 \%$ of body composition [1]. Physical activity greatly affects all components of physical fitness, aerobic exercise that is done will increase cardiorespiration endurance can reduce body fat [12]. Increased blood pressure in less trained people than in untrained people, the effect of breathing exercises on the breasts including expanding the area occurs during growth, but not in adulthood. The number of breaths per minute is reduced, trained people breathe 6 to 8 times per minute, whereas in untrained people as much as 18 to 20 times per minute. Deeper breathing with diaphragms. In people who are not trained the diaphragm moves very little. In doing the same work, trained individuals breathe in smaller amounts of air, and take in more oxygen than untrained individuals.

\section{Conclusions}

Research conducted to determine the fitness results of the Pekalongan City Basketball Index with the Harvard Step Test and Cooper Test methods is one method that can be used to measure the fitness of an athlete. From the initial measurements before the two methods were given, the condition of the athletes was at a limit that still needed to be improved. This can be seen from the ability during training and the initial measurement results, after adding training activities using the Harvard Step Test and Cooper Test methods, it can be concluded that the training results show a significant increase in the physical abilities of the basketball athlete. The value obtained for each athlete increases, with these results expected to help a trainer to further improve training and for an athlete to be able to continue to improve endurance. With better or increased endurance will reduce the risk of collapse in athletes caused by the inability to accept the portion of the exercise or compete. 


\section{References}

[1] Bandyopadhyay A. Validity of Cooper's 12-minute run test for estimation of maximum oxygen uptake in male university students. Biol. Sports. 2105; 58(2): 59-63.

[2] Cao ZB, Miyatake N, Aoyama T, Higuchi M, Tabata I. Prediction of Maximal Oxygen Uptake From a 3-Minute Walk Based on Gender, Age, and Body Composition. J. Phys. Act. Health. 2013; 10:280-7.

[3] Sunil KR, Das, S.K. and Mahapatra, S. Determination of physical fitness index (PFI) with modified Harvard Step Test (HST) in young men and women. Ind. J. Physiol and Allied Sci. 1993; 47(2): 73-76.

[4] Darmesh P. Study of Physical Fitness Index Using Modified Harvard Step Test in Relation with Gender in Physiotherapy Students. IJSR. 2015; 4(7):1215-1217.

[5] Fletcher GF, Ades PA, Arena R, Balady GJ, Bittner VA. Exercise standards for testing and training: a scientific statement from the American Heart Association. Circulation. 2013; 128:873-934.

[6] Myers J, Prakash M, Froelicher V Do D. Exercise capacity and mortality among men referred for exercise testing. N Engl J Med. 2002; 346:793-801.

[7] The Impact of Physical Activity (Running) on Cardiovascular Outcomes, https://www.acc.org/latest-in-cardiology/articles/2015/05/11/07/27/leisure-time-runningreduces-all-cause-and-cardiovascular-mortality-risk, last accessed 25 June 2020.

[8] Golbidi S, Laher I. Exercise and the Cardiovascular System. Cardiol. Res. Pract. 2012; 2012:1-15.

[9] Suharjana. 2013. Kebugaran Jasmani. Yogyakarta: Jogja Global Media; 2013.

[10] Exercise Physiology, https://emedicine.medscape.com/article/88484-overview, last accessed 25 June 2020.

[11] Sharkey BJ. Kebugaran \& Kesehatan. Jakarta: Raja Grafindo Persada; 2003.

[12] Shashiala L, Geetanjali H., Banner R., Sanjeev K., Natraj SM. Efficiency of recovery pulse rate as an index of physical fitness. JLS. 2014. 4(2):216-219. 